\title{
Significance of Macrophage Subtypes in the Peripheral Blood of Children with Systemic Juvenile Idiopathic Arthritis
}

Dan Feng $\cdot$ Wen-Yan Huang $\cdot$ Xiao-Ling Niu $\cdot$ Sheng Hao $\cdot$

Li-Ning Zhang $\cdot$ Yu-Jie $\mathrm{Hu}$

Received: September 4, 2021 / Accepted: October 5, 2021 / Published online: October 25, 2021

(C) The Author(s) 2021

\section{ABSTRACT}

Introduction: Symptomatic juvenile idiopathic arthritis (sJIA) is an autoinflammatory disease, and monocytes/macrophages play an important role. However, which macrophage subtype plays a major role in different stages of sJIA is still unclear. This study aimed to explore macrophage subtypes in different stages of sJIA.

Methods: Twenty-two children with sJIA who were followed up at Shanghai Children's Hospital from January 2018 to December 2020 were enrolled in this study. sJIA children were divided into an activity group $(n=12)$ and an inactivity group $(n=10)$. In the activity group, subjects with newly diagnosed sJIA and untreated were included; in the inactivity group, subjects with inactive sJIA meeting the 2011 ACR criteria for sJIA were recruited. Ten children with orthostatic proteinuria served as controls. Peripheral blood was collected. Flow cytometry was performed to detect macrophage subtypes: $\mathrm{M} 1 \quad\left(\mathrm{CD} 14^{+} \mathrm{CD} 86^{+} \mathrm{CD} 80^{+}\right), \quad \mathrm{M} 2 \mathrm{a}$ $\left(\mathrm{CD} 14^{+} \mathrm{CD} 206^{+} \mathrm{CD} 301^{+}\right), \mathrm{M} 2 \mathrm{~b}\left(\mathrm{CD} 14^{+} \mathrm{CD} 206^{+}\right.$ $\left.\mathrm{CD} 6^{+}\right)$and $\mathrm{M} 2 \mathrm{c}\left(\mathrm{CD} 14^{+} \mathrm{CD} 206^{+} \mathrm{CD} 163^{+}\right)$, and the contents of cytokines were also examined,

D. Feng · W.-Y. Huang $(\bowtie) \cdot$ X.-L. Niu · S. Hao ·

L.-N. Zhang · Y.-J. Hu

Department of Nephrology and Rheumatology,

Shanghai Children's Hospital, Shanghai Jiao Tong

University, 355 Luding Road, Shanghai 200062,

People's Republic of China

e-mail: huangwenyan@sjtu.edu.cn including interleukins (IL) (IL-1 $\beta$, IL-2, IL-4, IL5, IL-6, IL-8, IL-10 and IL-17), interferon- $\alpha$, interferon- $\gamma$, and tumor necrosis- $\alpha$.

Results: M1 marker CD80 and M2 marker CD163, CD301 were highly expressed in children with active sJIA. The majority of macrophages were M1 and M2a in the activity group $(P<0.05)$. In the inactivity group, M2 tended to polarize into M2b and M2c $(P<0.05)$. IL-6 significantly increased in the activity group $(P<0.05)$, while IL-10, IL-4 and IL-17 markedly increased in the inactivity group $(P<0.05)$.

Conclusions: In the active SJIA, M1 activation promotes inflammation, while $\mathrm{M} 2 \mathrm{a}$ rapidly responds to inhibit inflammation; in the inactive sJIA, M2b and M2c play a major role in inhibiting inflammation.

Keywords: Cytokines; Macrophage subtypes; Symptomatic juvenile idiopathic arthritis 


\section{Key Summary Points}

Macrophages play an important role in the occurrence and development of sJIA. In the active stage of sJIA, M1 activation promotes inflammation, while M2a rapidly acts to suppress inflammation.

In the inactive stage, M2b and M2c play a major role in inhibiting inflammation.

Different macrophage subtypes are related to cytokines such as IL-6, IL-10, IL-4, and IL-17, and both of them participate in the development and remission of sJIA.

\section{INTRODUCTION}

Systemic juvenile idiopathic arthritis (sJIA) is a special subtype of juvenile idiopathic arthritis. Compared with other subtypes, sJIA is more comparable to autoinflammatory disease. In addition to the manifestations of arthritis, sJIA is characterized by systemic symptoms such as fever and rash and prone to macrophage activation syndrome [1-4]. Recent studies have shown that the activation of monocytes/macrophages plays an important role in the pathogenesis of sJIA [5-7]. Various types of macrophages can be present in different cytokine microenvironments [8]. According to the differences in the identified molecules on the cells and cell functions, macrophages are mainly divided into two types: M1 macrophages (classically activated macrophages) and M2 macrophages (alternatively activated macrophages) [9]. M1 macrophages can cause inflammation and produce pro-inflammatory cytokines such as tumor necrosis factor (TNF)- $\alpha$, interleukin (IL)-1 $\beta$, IL-6, IL-12, CXCL9 and CXCL10, and low levels of IL-10 regulate and promote Th1-type immune response [10, 11]. Cytokines such as IL-10, IL-4, and IL-13 can induce polarization into M2 macrophages. Functionally, M2 macrophages can inhibit the inflammatory response, remove debris and apoptotic cells, promote tissue repair and wound healing, improve immune regulation, and promote angiogenesis and fibrosis $[12,13]$. Recently, studies have found that M2 macrophage-related genes are expressed in the monocytes of sJIA children [5], such as CD163, Arg1, and CCl2. However, further studies on macrophages have found that M2 macrophages under a specific environment can be further divided into different subtypes, namely, M2a, M2b, and M2c. Different subtypes play different roles in various stages of this disease. For example, M2a participates in Th2 reaction and allergic reactions and can kill parasites, while $\mathrm{M} 2 \mathrm{~b}$ is involved in immune regulation. M2c has a de-priming effect and can secrete various antiinflammatory cytokines, which are mainly involved in tissue remodeling and matrix deposition [14-16]. However, the role of macrophage subtypes in the pathogenesis of sJIA has not been thoroughly investigated so far. This study aims to examine the macrophage subtypes, surface markers, and cytokines secreted by macrophages in children with sJIA at different stages.

\section{METHODS}

\section{Patients}

This study enrolled 22 children with sJIA who were followed up at Shanghai Children's Hospital from January 2018 to December 2020. The sJIA was diagnosed based on the International League of Associations for Rheumatology (ILAR) 2001 JIA criteria [17]. sJIA children were divided into an activity group $(n=12)$ and an inactivity group $(n=10)$. In the activity group, children who were newly diagnosed with sJIA and did not receive treatment before study were recruited. In the inactivity group, children were diagnosed with sJIA in inactive phase according to the 2011 ACR criteria (no joints with active arthritis; no fever, rash, serositis, splenomegaly, or generalized lymphadenopathy attributable to JIA; no active uveitis to be defined; erythrocyte sedimentation rate (ESR) or C-reactive protein (CRP) level within normal limits in the laboratory where tested (if both are tested, both must 
be normal) and physician's global assessment of disease activity score of best possible on the scale used [18]. In the inactivity group, children were treated with prednisone, methotrexate, and IL- 6 receptor antagonist to improve symptoms. Prednisone treatment was stopped 5-12 months ago at the analysis of macrophage subtypes, and methotrexate and IL- 6 receptor antagonists were still used for maintenance treatment. In the control group, ten children with orthostatic proteinuria were included.

This study was approved by the Ethics Committee of Shanghai Children's Hospital (No. 2017R026-F01) and informed consent was obtained by the patient's parents. This study was performed in accordance with the Helsinki Declaration of 1964 and its later amendments.

\section{Detection of Macrophage Subtypes}

Flow cytometry was performed to detect monocytes/macrophages in the peripheral blood of children in two groups. Cells were subjected to incubation with CD14-FITC, CD80APC, CD86-PE/Cyanine7, CD301-PE, CD206Alexa Flour ${ }^{\circledR} 700$, and CD163-Brilliant Violet $421^{\mathrm{TM}}$. All steps were performed according to the manufacturer's instructions. All reagents were from Biolegend, and the Cytoflex S flow cytometer was used.

We defined $\mathrm{CD} 14^{+} \mathrm{CD} 86^{+} \mathrm{CD} 80^{+}$as $\mathrm{M} 1$, $\mathrm{CD}_{14}{ }^{+} \mathrm{CD}_{206}{ }^{+} \mathrm{CD} 01^{+}$as $\mathrm{M} 2 \mathrm{a}, \mathrm{CD} 14^{+}$

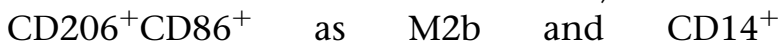
$\mathrm{CD} 206^{+} \mathrm{CD}_{163^{+}}$as M2c.

\section{Detection of cytokines}

The cytokines (IL- $1 \beta$, IL-2, IL-4, IL-5, IL-6, IL-8, IL-10, IL-17, IFN- $\alpha$, IFN- $\gamma$, and TNF- $\alpha$ ) in the peripheral blood were detected by flow cytometry. All steps were performed according to the manufactures' instructions. Reagents were obtained from RaiseCare (China).

\section{Statistical Analysis}

Statistical Product and Service Solutions (SPSS) version 19.0 (SPSS Inc., Chicago, IL, USA) was used for statistical analysis. Quantitative data with normal distribution were analyzed by analysis of variance, while qualitative data without normal distribution are expressed as median (interquaternary interval). A non-parametric Kruskal-Wallis test was used for comparison of other continuous variables. A value of $P<0.05$ was considered statistically significant. Flow cytometry data were analyzed with FlowJo10. All graphics were created using GraphPad Prism 8.0.

\section{RESULTS}

\section{Characteristics of Patients on Macrophage Subtype Assay}

There were 32 patients in the present study, including 17 males and 15 females. In the activity group, there were 12 patients (seven males, five females, male:female $=1.40: 1$ ); the mean age was $8.38 \pm 2.77$ years; the average levels of ESR and CRP were $94.83 \pm 15.78 \mathrm{~mm} /$ $\mathrm{h}$ and $111.53 \pm 25.23 \mathrm{mg} / \mathrm{l}$, respectively. In the inactivity group, there were ten patients (four males, six females, male:female $=0.66: 1$ ); the mean age was $9.91 \pm 2.16$ years, and the levels of ESR and CRP returned to normal. In the control group, there were ten subjects (six males, four females, male:female $=1.50: 1$ ); the mean age was $10.65 \pm 2.13$ years, and the levels of ESR and CRP were normal. The results are shown in Table 1.

\section{Phenotype of CD14 ${ }^{+}$Monocyte/ Macrophage Subsets in sJIA Children}

Flow cytometry was performed to detect the surface markers of M1 and M2 in the CD14 ${ }^{+}$ mononuclear subsets based on the mean fluorescence intensity (MFI). Results showed that the expression of M1 marker CD80 in the activity group was significantly higher than in the inactivity group and control group (Fig. 1A). CD86 expression was comparable among three groups (Fig. 1B). CD206, CD163, and CD301 served as markers of M2. Results showed that CD163 expression in the activity group was significantly higher than in the control group, 
and CD163 expression tended to be normal after remission of sJIA (Fig. 1C). The MFI of CD301 in the activity group was significantly higher than in the inactivity group and control group (Fig. 1D), but no significant difference was found in CD206 expression among three groups (Fig. 1E).

\section{Difference in Macrophage Subtypes in sJIA Children}

First, we gated monocytes in a forward scatter/ sideward scatter (FSC/SSC) dot plot. Second, we gated $\mathrm{CD}_{14}{ }^{+}$monocytes within monocytes. Finally, we gated M1, M2a, M2b, and M2c using isotype controls within $\mathrm{CD} 14^{+}$monocytes. Proportions of M1 and M2a in the activity group were higher than in the control group (Fig. 2A, B), indicating that M1 and M2a played an important role in the activity of sJIA. In the activity group, the proportions of M2 subtypes in the total M2 were significantly different (Fig. 2C): the proportions of both M2a and M2b were higher than that of M2c, but no difference was found between M2a and M2b. In the inactivity group, $\mathrm{M} 2 \mathrm{a}$ decreased significantly as compared to M2b and M2c, and the M2 subtypes showed the same trend in the inactivity group and control group.
Fig. 1 Mean fluorescence intensity of M1-and M2-related surface markers. A Expression of M1-related marker CD80 in sJIA children and controls. B Expression of M1-related marker CD86 in sJIA children and controls. C Expression of M2-related marker CD163 in sJIA children and controls. D Expression of M2-related marker CD301 in sJIA children and controls. E Expression of M2-related marker CD206 in sJIA children and controls. F FACS histograms of M1- and M2-related surface markers. Activity group, $n=12$; inactivity group, $n=10$; control group, $n=10 .{ }^{*} P<0.05{ }^{* *} P<0.01$

\section{Cytokines in the Peripheral Blood of sJIA Children}

Cytokines (IL-1 $\beta$, IL-2, IL-4, IL-5, IL-6, IL-8, IL10 , IL-17, IFN- $\alpha$, IFN- $\gamma$, and TNF- $\alpha$ ) in the blood were detected by flow cytometry (Table 2). Results showed that the IL-6 in the activity group was significantly higher than in the inactivity and control groups. IL-6 was still higher than normal in the inactivity group. Interestingly, IL-4, IL-10, and IL-17 markedly increased in the inactivity group, while other cytokines were comparable among three groups.

\section{DISCUSSION}

Macrophages are an important component of innate immunity and play a central role in the inflammation and host defense. In response to

Table 1 Characteristics of patients at the time of macrophage subtyping ( $n$ or mean \pm SD)

\begin{tabular}{llll}
\hline Characteristics & Activity group & Inactivity group & Control group \\
\hline Number of patients & 12 & 10 & 10 \\
Gender & & & \\
Male & 7 & 4 & 6 \\
Female & 5 & 6 & 4 \\
Age (years) & $8.38 \pm 2.77$ & $9.91 \pm 2.16$ & $10.65 \pm 2.13$ \\
ESR $(\mathrm{mm} / \mathrm{h})$ & $94.83 \pm 15.78$ & $2.10 \pm 0.13$ & $9.20 \pm 7.26$ \\
CRP $(\mathrm{mg} / \mathrm{l})$ & $111.53 \pm 25.23$ & $\leq 5$ & $\leq 5$ \\
\hline
\end{tabular}

ESR erythrocyte sedimentation rate, $C R P \mathrm{C}$-reactive protein 

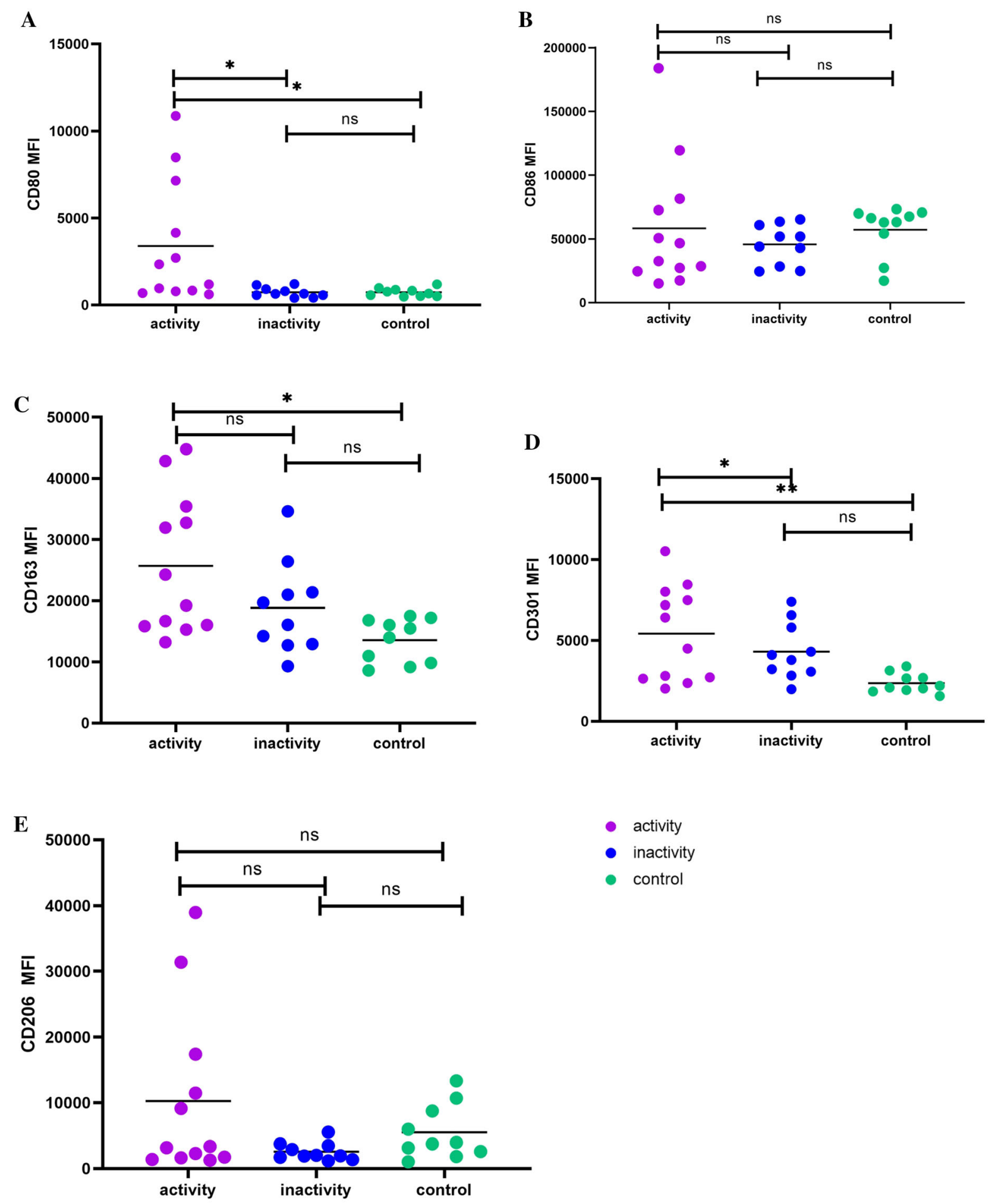

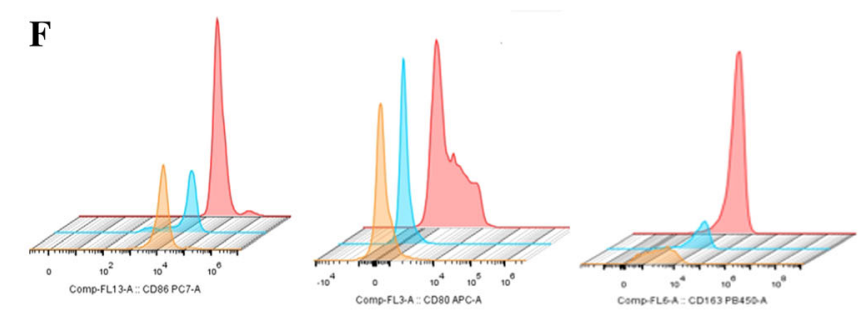
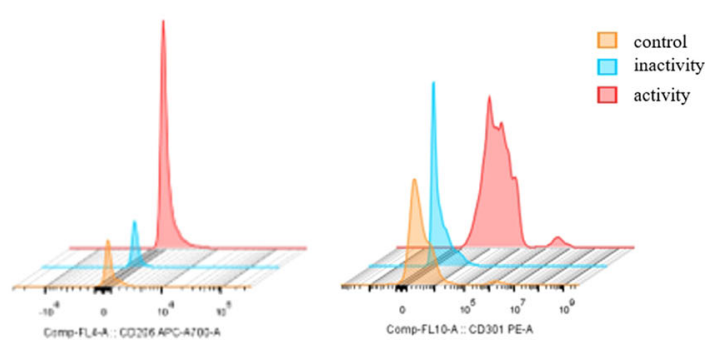

Fig. 1 continued

various environmental factors (e.g., microbial products, damaged cells, and activated lymphocytes) or different pathophysiological conditions, macrophages transform into different functional phenotypes as classically activated macrophages (M1) and alternatively activated macrophages (M2) $[8,19]$. Recent studies have classified M2 into M2a, M2b, and M2c according to the induced molecules and transcription products $[11,12]$. At present, numerous studies have shown that macrophages play an important role in the occurrence and development of sJIA, but the role of different subtypes of macrophages at different stages of sJIA is still poorly understood.

It has been reported that the M1 macrophages highly express CD80, CD86, and MHCII and can secret IL-1, IL- 6 , IL-12, and TNF- $\alpha$ $[13,20]$. M2a macrophages are characterized by CD206, CD301, CD200R, Arg1, and other molecules, and can secrete IL-10 and TGF- $\beta$ at the same time [21, 22]. The signature molecules of M2b macrophages include CD86, CD206, and MHCII, and they can secrete IL-10, TNF- $\alpha$, IL-1 $\beta$, and IL-6 [15, 23]. Markers of M2c include CD163, CD206, MERTK, and CXCL13, and these cells can secrete IL-10 and TGF- $\beta$ and are simultaneously activated by IL-10 and glucocorticoids [12, 14, 24].

In this study, the characteristic surface markers were detected aiming to subtype macrophages. Our results showed that the expression of CD80, a surface marker of M1, increased at the active stage of sJIA, while CD86 expression was comparable among three groups, which was consistent with the results of Macaubas et al. [5]. CD80 and CD86 are important molecules that can provide synergistic stimulus signals, which can bind to ligands CD28 and CTLA-4 on the T lymphocytes to provide synergistic signals, leading to the activation of $\mathrm{T}$ lymphocytes [25, 26]. At present, the CD80 antagonist abatacept has been used in the treatment of sJIA in children achieving favorable efficacy [27]. Our results indicate that CD80 antagonists may also be applied in the clinical treatment of sJIA in children. Our study also revealed that M2 markers CD163 and CD301 were highly expressed in the activity group as compared to the control group, but CD206 expression was comparable among the three groups. A variety of studies have shown that CD163 expression significantly increases in case of active sJIA, which is consistent with our findings $[5,28]$. CD163 is a scavenger receptor with mononuclear macrophage specificity. It is highly expressed on mature macrophages (mainly M2 macrophages) in various tissues. Recent studies have shown that CD163 is highly expressed mainly in M2c macrophages and plays an antiinflammatory role in sJIA. CD206 is a scavenger receptor and a marker of M2 macrophages $[29,30]$. Macaubas et al. also found that CD206 expression increased in the peripheral blood of active sJIA patients [5]. However, our study indicated the CD206 expression increased in the activity group as compared to the inactivity group and control group, although there was no significant difference, which might be related to the small sample size. CD301, also known as macrophage galactose-calcium lectin, is a C-type lectin receptor that can be highly expressed in macrophages after IL- 4 treatment [31]. It is related to the uptake of glycoprotein, and the interaction between immune cells and 

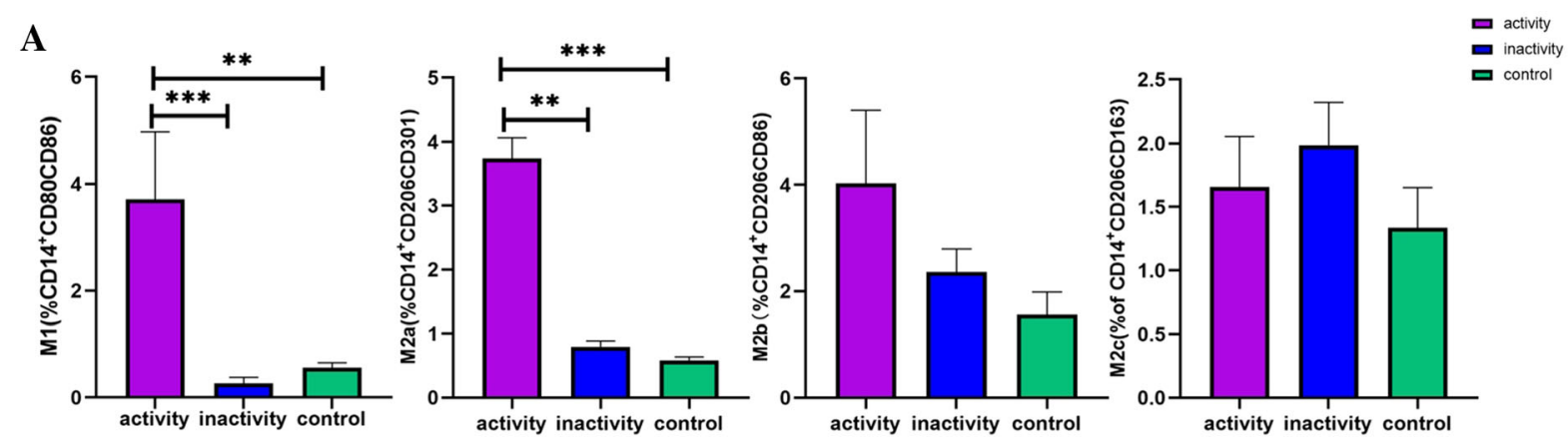

B B
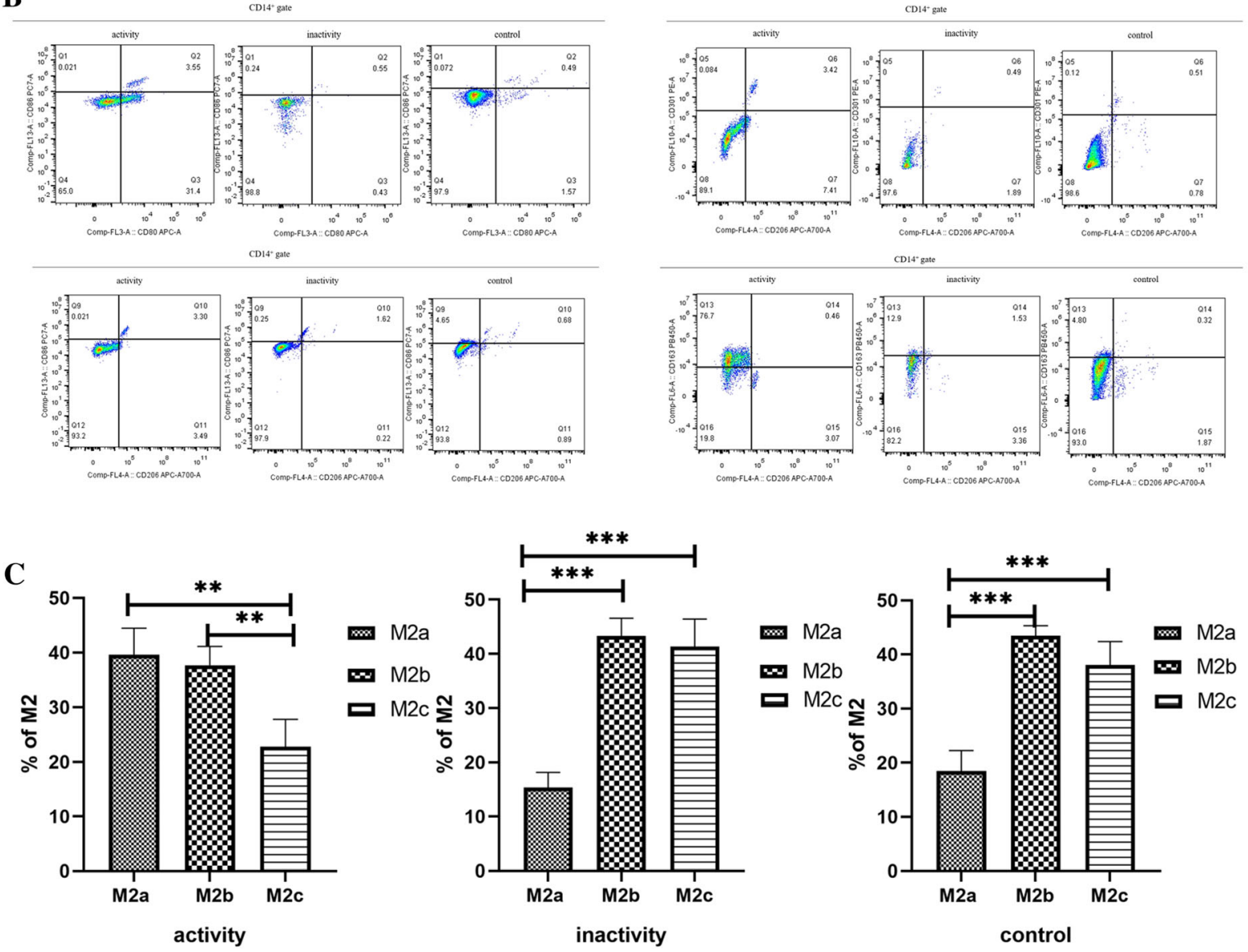

Fig. 2 Subtypes of macrophages in children with sJIA. A The proportions of $\mathrm{M} 1, \mathrm{M} 2 \mathrm{a}, \mathrm{M} 2 \mathrm{~b}$, and $\mathrm{M} 2 \mathrm{c}$ in different stages of sJIA children and controls. B Representative flow chart of the proportions of M1, M2a, M2b, and $\mathrm{M} 2 \mathrm{c}$ in different stages of sJIA children and controls.

pathogen recognition [32], but its expression in SJIA has not been reported yet. To our knowledge, this study for the first time reported that CD301 expression significantly increased in sJIA
C M2a, M2b, and M2c accounted for the proportion of total M2 in the same period of sJIA children and controls. Activity group, $n=12$; inactivity group, $n=10$; control group, $n=10 .{ }^{*} P<0.05{ }^{* *} P<0.01{ }^{* * *} P<0.001$

in active phase, but the specific role of CD301 in sJIA remains to be further evaluated.

At the active stage, sJIA is mainly manifested by the massive secretion of inflammatory 
Table 2 The expression of cytokines in the plasma of sJIA children (mean \pm SD; pg/ml)

\begin{tabular}{lccc}
\hline Cytokines & Activity group & Inactivity group & Control group \\
\hline IL-6 & $229.96 \pm 345.41^{* \#}$ & $17.033 \pm 9.56^{*}$ & $0.14 \pm 0.05$ \\
IL-5 & $8.10 \pm 5.12$ & $5.44 \pm 4.76$ & $0.34 \pm 0.77$ \\
IFN- $\alpha$ & $3.61 \pm 2.93$ & $0.1 \pm 0.15$ \\
IL-2 & $4.14 \pm 1.35$ & $4.10 \pm 3.56$ & $0.18 \pm 0.27$ \\
IL-10 & $3.57 \pm 11.40$ & $2.13 \pm 1.56^{*}$ & $0.16 \pm 0.2$ \\
IFN- $\gamma$ & $2.19 \pm 5.45$ & $8.552 \pm 7.56$ & $23.778 \pm 23.13$ \\
IL-8 & $44.7 \pm 29.99$ & $8.78 \pm 8.91$ & $65.10 \pm 47.45$ \\
IL-17 & $65.11 \pm 63.18$ & $2.46 \pm 0.63^{*}$ & $0.17 \pm 0.07$ \\
IL-1 $\beta$ & $7.26 \pm 6.54$ & $13.03 \pm 4.20$ & $0.53 \pm 0.25$ \\
IL-4 & $38.44 \pm 33.65$ & $2.03 \pm 1.45^{*}$ & $0.27 \pm 0.37$ \\
TNF- $\alpha$ & $1.61 \pm 3.35$ & $6.85 \pm 5.73$ & $0.28 \pm 0.46$ \\
\hline
\end{tabular}

Notes: activity group, $n=12$; inactivity group, $n=10$; control group, $n=10 .{ }^{*} P<0.05$ vs. control group; ${ }^{\#} P<0.05$ vs. inactivity group

cytokines and outbreak of inflammatory response $[1,3,33]$, while $\mathrm{M} 1$, as a typical macrophage, can promote the inflammation and accelerate the degradation of extracellular matrix and apoptosis by secreting large amounts of cytokines such as TNF- $\alpha$, IL- 6 , IL- $1 \beta$, IL-23, etc. In this study, M1 macrophages increased in the activity group $(P<0.05)$, and the M1 macrophages in the inactivity group were similar to those in the control group. However, M2a macrophages increased in the active stage of sJIA, which has not been reported yet. Many studies have shown that monocytes in the peripheral blood of children with untreated active sJIA express molecules related to M2 macrophages, such as CD163, Arg1, IL10, MS4A4a, and CCL2 [5, 6, 34]. However, the specific subtype of macrophages that play a major role in the active phase of sJIA is not yet known. Haribhai et al. found that implantation of M2a macrophages into mice with inflammatory bowel disease served as an immune adjuvant for cell transfer immunotherapy to promote the directed and consistent expansion of the iTreg-Th17 cell axis and reconstruct mucosal immunity [35]. Therefore, we hypothesized that M2a macrophages were stimulated by various inflammatory cytokines at an early stage of sJIA to produce a rapid immune response and thereby inhibit the inflammatory cytokine storm.

In this study, sJIA children were mainly treated with prednisone, methotrexate, and IL-6 receptor antagonist (tocilizumab), aiming to improve symptoms. In the present study, prednisone treatment was discontinued about half a year ago in the inactive group, but they were still taking methotrexate orally, and the time interval to the last dose of tocilizumab was 2-6 weeks. In the inactivity group, levels of M1 and M2a basically returned to normal. Among the M2 subtypes, the macrophages in children with sJIA in the remission stage were mainly $\mathrm{M} 2 \mathrm{~b}$ and M2c, and the percentage of M2a significantly decreased $(P<0.05)$, which was consistent with the observations in the control group. However, in children with active sJIA, $\mathrm{M} 2 \mathrm{c}$ in the M2 subtype reduced, and M2a and M2b were the dominant subtypes, suggesting that M2b and M2c play a decisive role in the late remission of sJIA. There is evidence showing that both $\mathrm{M} 2 \mathrm{~b}$ and $\mathrm{M} 2 \mathrm{c}$ have immunoregulatory effects, but whether the decrease in the 
$\mathrm{M} 2 \mathrm{a}$ and the increase in the $\mathrm{M} 2 \mathrm{c}$ are related to tocilizumab treatment remains to be evaluated.

To further investigate the role of macrophage subtypes at different stages of sJIA and the potential mechanism, cytokines were detected and analyzed in the above three groups. IL-6 increased significantly in the activity group, but decreased significantly in the inactive group as compared to the activity group, but it failed to completely return to normal, while other cytokines did not significantly increase in the activity group. Studies have shown that sustained high IL-6 level is not only associated with the degree of joint damage and osteoporosis but also with the clinical manifestations such as fever, increased platelet consumption, anemia, and growth retardation in sJIA children [36, 37]. IL-6 can bind to the IL6 receptor and glycoprotein 130 to stimulate the JAK-STAT signaling pathway, thus activating the inflammatory response [38]. Moreover, IL- 6 can be produced by the M1 macrophages. Our study also indicated that IL-4, IL-10, and IL17 increased in the inactivity group as compared to the control group. IL-4 is mainly secreted by Th2 cells and can induce the differentiation of macrophages into $\mathrm{M} 2 \mathrm{a}$, which is involved in the inhibition of inflammation $[12,13]$. However, few recent studies have evaluated the role of IL-4 in sJIA. Some studies have shown that the expression of IL-10 in the plasma and synovial fluid of active sJIA children is higher than in those with inactive sJIA [39]. However, other studies have indicated that expression of IL-10 in active sJIA children is lower than in those with inactive sJIA, suggesting that IL-10 can act as a protective anti-inflammatory factor and antagonize the destruction of cartilage and bone tissues in the pathogenesis of sJIA. In our study, IL-10 expression was elevated in the inactivity group, which may exert a protective effect. It has been reported that IL-10 can be secreted by M2a, $\mathrm{M} 2 \mathrm{~b}$, and M2c, has a wide range of anti-inflammatory effects, and can induce the formation of M2c, which may attribute to the increase of M2c in the remission stage of sJIA in the present study. The inflammatory cytokine IL-17 is secreted by Th17 cells, and can promote the occurrence and development of sJIA. Our results showed that IL-17 level was significantly higher in the active stage than in the inactivity stage, and there was a positive correlation between IL17 and IL-6. IL-17 and IL-6 play a synergistic role in the pathogenesis of sJIA $[40,41]$. In our study, the IL-17 level increased in both active and inactive phases of sJIA, and in the inactivity group, it was significantly different from that in the control group $(P<0.05)$.

There were still limitations in the present study. First, the sample size was small, and more studies with large sample size are needed to confirm our findings in the future. In our study, children with orthostatic proteinuria were included as controls, although no study has reported the involvement of macrophages and cytokines in the pathogenesis of orthostatic proteinuria. In addition, the JIA subgroup distribution was not homogeneous in the present study.

\section{CONCLUSIONS}

Macrophages play an important role in the occurrence and development of sJIA. In the active stage of sJIA, M1 activation promotes inflammation, while $\mathrm{M} 2 \mathrm{a}$ rapidly acts to suppress inflammation. In the inactive stage, $\mathrm{M} 2 \mathrm{~b}$ and $\mathrm{M} 2 \mathrm{c}$ play a major role in inhibiting inflammation. Different macrophage subtypes may be related to the production of different cytokines (such as IL-6, IL-10, IL-4, and IL-17), and both cells and cytokines participate in the development and remission of sJIA.

\section{ACKNOWLEDGEMENTS}

We thank the participants of the study.

\section{Declarations}

Funding This study was supported by the Youth Program Foundation by Shanghai Municipal Commission of Health and Family Planning (No. 20174Y0086), Natural Science Foundation by the Shanghai Municipal Commission of Science and Technology (No. 19ZR1442300), and Clinical Research 
Foundation by the Shanghai Children's Hospital (No. 2020YGZQ02). The journal's Rapid Service Fee was funded by the authors.

Authorship All named authors meet the International Committee of Medical Journal Editors (ICMJE) criteria for authorship for this article, take responsibility for the integrity of the work as a whole, and have given their approval for this version to be published.

Authorship Contributions DF performed flow cytometry, the statistical analysis, and drafted the article. XLN and SH was responsible for inclusion of children. LNZ and $\mathrm{YJH}$ detected cytokines. WYH is responsible for the study design and coordination. All authors have read and approved the final manuscript.

Disclosures Dan Feng, Wen-Yan Huang*, Xiao-Ling Niu, Sheng Hao, Li-Ning Zhang, and Yu-Jie $\mathrm{Hu}$ have nothing to disclose.

Compliance with Ethics Guidelines This study was approved by the Ethics Committee of Shanghai Children's Hospital (No. 2017R026F01), and informed consent was obtained by the patient's parents. This study was performed in accordance with the Helsinki Declaration of 1964 and its later amendments.

Data Availability The datasets generated during and analyzed during the current study are available from the corresponding author on reasonable request.

Open Access. This article is licensed under a Creative Commons Attribution-NonCommercial 4.0 International License, which permits any non-commercial use, sharing, adaptation, distribution and reproduction in any medium or format, as long as you give appropriate credit to the original author(s) and the source, provide a link to the Creative Commons licence, and indicate if changes were made. The images or other third party material in this article are included in the article's Creative Commons licence, unless indicated otherwise in a credit line to the material. If material is not included in the article's Creative Commons licence and your intended use is not permitted by statutory regulation or exceeds the permitted use, you will need to obtain permission directly from the copyright holder. To view a copy of this licence, visit http://creativecommons.org/licenses/by$\mathrm{nc} / 4.0 /$.

\section{REFERENCES}

1. Albaker AR. Current review of systemic juvenile idiopathic arthritis: What do paediatricians need to know? Open J Pediatr. 2020;10:33.

2. Cimaz R. Systemic-onset juvenile idiopathic arthritis. Autoimmun Rev. 2016;15:931-4.

3. Russo RA, Katsicas MM. Patients with very earlyonset systemic juvenile idiopathic arthritis exhibit more inflammatory features and a worse outcome. J Rheumatol. 2013;40:329-34.

4. Barut K, Adrovic A, Sahin S, et al. Prognosis, complications and treatment response in systemic juvenile idiopathic arthritis patients: a single-center experience. Int J Rheum Dis. 2019;22(9):1661-9.

5. Macaubas C, Nguyen KD, Peck A, et al. Alternative activation in systemic juvenile idiopathic arthritis monocytes. Clin Immunol. 2012;142:362-72.

6. Ogilvie EM, Khan A, Hubank M, Kellam P, Woo P. Specific gene expression profiles in systemic juvenile idiopathic arthritis. Arthritis Rheum. 2007;56: 1954-65.

7. Schulert GS, Fall N, Harley JB, et al. Monocyte microRNA expression in active systemic juvenile idiopathic arthritis implicates microRNA-125a-5p in polarized monocyte phenotypes. Arthritis Rheumatol. 2016;68:2300-13.

8. Funes SC, Rios M, Escobar-Vera J, Kalergis AM. Implications of macrophage polarization in autoimmunity. Immunology. 2018;154:186-95.

9. Italiani $\mathrm{P}$, Boraschi D. From monocytes to M1/M2 macrophages: phenotypical vs. functional differentiation. Front Immunol. 2014;5:514.

10. Fukui S, Iwamoto N, Takatani A, et al. M1 and M2 monocytes in rheumatoid arthritis: a contribution of imbalance of M1/M2 monocytes to osteoclastogenesis. Front Immunol. 2017;8:1958.

11. Mills CD. Anatomy of a discovery: M1 and M2 macrophages. Front Immunol. 2015;6:212.

12. Rőszer T. Understanding the mysterious M2 macrophage through activation markers and effector 
mechanisms. Mediators Inflamm. 2015;2015: 816460.

13. Sica A, Mantovani A. Macrophage plasticity and polarization: in vivo veritas. J Clin Invest. 2012;122: 787-95.

14. Chen Z, Dong F, Lu J, et al. Polarized M2c macrophages have a promoting effect on the epithelial-tomesenchymal transition of human renal tubular epithelial cells. Immunobiology. 2018;223:826-33.

15. Wang LX, Zhang SX, Wu HJ, Rong XL, Guo J. M2b macrophage polarization and its roles in diseases. J Leukoc Biol. 2019;106:345-58.

16. Zhang MZ, Wang X, Wang Y, et al. IL-4/IL-13-mediated polarization of renal macrophages/dendritic cells to an M2a phenotype is essential for recovery from acute kidney injury. Kidney Int. 2017;91: $375-86$.

17. Petty RE, Southwood TR, Manners P, et al. International league of associations for rheumatology classification of juvenile idiopathic arthritis: second revision, Edmonton, 2001. J Rheumatol. 2004;31(2):390-2.

18. Carol A, Wallace, Edward H, et al. American College of Rheumatology provisional criteria for defining clinical inactive disease in select categories of juvenile idiopathic arthritis. Arthritis Care Res. 2011;63(7):929-36.

19. Murray PJ. Macrophage polarization. Annu Rev Physiol. 2017;79:541-66.

20. Martinez FO, Gordon S. The M1 and M2 paradigm of macrophage activation: time for reassessment. F1000Prime Rep. 2014;6:13.

21. Chistiakov DA, Bobryshev YV, Nikiforov NG, Elizova NV, Sobenin IA, Orekhov AN. Macrophage phenotypic plasticity in atherosclerosis: the associated features and the peculiarities of the expression of inflammatory genes. Int J Cardiol. 2015;184: 436-45.

22. Forbes SJ, Rosenthal N. Preparing the ground for tissue regeneration: from mechanism to therapy. Nat Med. 2014;20:857-69.

23. Edwards JP, Zhang X, Frauwirth KA, Mosser DM. Biochemical and functional characterization of three activated macrophage populations. J Leukoc Biol. 2006;80:1298-307.

24. Mosser DM, Edwards JP. Exploring the full spectrum of macrophage activation. Nat Rev Immunol. 2008;8:958-69.
25. Porciello N, Tuosto L. CD28 costimulatory signals in $\mathrm{T}$ lymphocyte activation: emerging functions beyond a qualitative and quantitative support to TCR signalling. Cytokine Growth Factor Rev. 2016;28:11-9.

26. Vasu C, Wang A, Gorla SR, Kaithamana S, Prabhakar BS, Holterman MJ. CD80 and CD86 C domains play an important role in receptor binding and co-stimulatory properties. Int Immunol. 2003;15:167-75.

27. Ruperto N, Lovell DJ, Quartier P, et al. Long-term safety and efficacy of abatacept in children with juvenile idiopathic arthritis. Arthritis Rheum. 2010;62:1792-802.

28. Thornton S, Tan R, Sproles A, et al. A multiparameter flow cytometry analysis panel to assess CD163 mRNA and protein in monocyte and macrophage populations in hyperinflammatory diseases. J Immunol. 2019;202:1635-43.

29. Aron-Wisnewsky J, Tordjman J, Poitou C, et al. Human adipose tissue macrophages: M1 and M2 cell surface markers in subcutaneous and omental depots and after weight loss. J Clin Endocrinol Metab. 2009;94:4619-23.

30. Murray PJ, Allen JE, Biswas SK, et al. Macrophage activation and polarization: nomenclature and experimental guidelines. Immunity. 2014;41: $14-20$.

31. Brooks PJ, Wang Y, Magalhaes MA, Glogauer M, McCulloch CA. CD301 mediates fusion in IL-4-driven multinucleated giant cell formation. J Cell Sci. 2020. https://doi.org/10.1242/jcs.248864.

32. van Kooyk Y, Ilarregui JM, van Vliet SJ. Novel insights into the immunomodulatory role of the dendritic cell and macrophage-expressed C-type lectin MGL. Immunobiology. 2015;220:185-92.

33. Mellins ED, Macaubas C, Grom AA. Pathogenesis of systemic juvenile idiopathic arthritis: some answers, more questions. Nat Rev Rheumatol. 2011;7:416-26.

34. Fall N, Barnes M, Thornton S, et al. Gene expression profiling of peripheral blood from patients with untreated new-onset systemic juvenile idiopathic arthritis reveals molecular heterogeneity that may predict macrophage activation syndrome. Arthritis Rheum. 2007;56:3793-804.

35. Haribhai D, Ziegelbauer J, Jia S, et al. Alternatively activated macrophages boost induced regulatory $\mathrm{T}$ and Th17 cell responses during immunotherapy for colitis. J Immunol. 2016;196:3305-17. 
36. Fonseca JE, Santos MJ, Canhão H, Choy E. Interleukin- 6 as a key player in systemic inflammation and joint destruction. Autoimmun Rev. 2009;8: 538-42.

37. Nishimoto $\mathrm{N}$, Kishimoto $\mathrm{T}$. Interleukin 6: from bench to bedside. Nat Clin Pract Rheumatol. 2006;2:619-26.

38. Kaur S, Bansal Y, Kumar R, Bansal G. A panoramic review of IL-6: Structure, pathophysiological roles and inhibitors. Bioorg Med Chem. 2020;28: 115327.

39. van den Ham HJ, de Jager W, Bijlsma JW, Prakken BJ, de Boer RJ. Differential cytokine profiles in juvenile idiopathic arthritis subtypes revealed by cluster analysis. Rheumatology (Oxford). 2009;48: 899-905.

40. Jiang H, Zhu M, Wang H, Liu H. Suppression of IncRNA MALAT1 reduces pro-inflammatory cytokines production by regulating miR-150-5p/ ZBTB4 axis through JAK/STAT signal pathway in systemic juvenile idiopathic arthritis. Cytokine. 2021;138: 155397.

41. Omoyinmi E, Hamaoui R, Pesenacker A, et al. Th1 and Th17 cell subpopulations are enriched in the peripheral blood of patients with systemic juvenile idiopathic arthritis. Rheumatology (Oxford). 2012;51:1881-6. 